\title{
Exploration of the Employment of Idea Training in Teaching Forehand Attack of Table Tennis
}

\author{
Chunxia Yang ${ }^{1}$ \\ ${ }^{1}$ Yunnan College of Tourism Vocation, Kunming, Yunnan, China
}

Keywords: representation phenomenon; table tennis; forehand attack teaching

\begin{abstract}
As an important event among sports items, table tennis could not only enrich daily life of people relieving them from pressure of work and study, but also strengthen their body. Forehand attack ball, also called near platform fast pumping, is one of table tennis attacks. However, there are several problems in actual forehand attack teaching, and under the educational reform, coach should transfer the traditional teaching model, applying idea training in forehand attack in table tennis teaching and thus combine teaching strategy with mental training that could improve whole effect of forehand attack teaching. This paper applies experimental inquiry method to explore application of idea training in forehand tables of table tennis, hoping it will offer valuable reference to table tennis teaching.
\end{abstract}

\section{Introduction}

There are many great table tennis players in our country, and in the world-class sports events, we can always get wonderful ranks, therefore, viewed as national sports, table tennis has consisted in public sports items as for many high schools and outdoor sports. Table tennis is not only testify technique movements of students, but also mental ability, the traditional forehand attack teaching could not meet developing demand of players, thus, under the background of educational reform, we could try to apply idea training into forehand attack teaching to form a stereotyped image while taking exercise, and then improve the teaching effect of forehand attack. From it, we could find that it has important meaning to explore the representation phenomenon in forehand attack training

\section{Concept and Function of Idea Training}

Idea training, or imagery training, psychological training as well, mainly means that players will perform and rebuild the past learning skills in the side of mind with consciousness, thus, the nature of this training is the mental process of trainers. In forehand attack teaching for table tennis, idea training has raised wide attention from educators and has been the main teaching method gradually, to apply idea training in table tennis with forehand attack teaching is to replay the existed picture of sports skill and scenes of forehand attack, and then understand and recreate, it is a set of mental process [1].

There are various function of idea training, it could show in specific as following: first, when applying it in forehand attack teaching of table tennis, trainers could enforce the cognitive consciousness of such movement through mental function, and therefore they could combine consciousness and training closely that fine the mistake between imagery movements and actuality, once students realize these problem and take timely responding measure to correct, they then could promote the normalization [2]. Apart from it, when applying such training in forehand attack of table tennis, it could raise a certain learning interest of trainers, because of the high difficulty of forehand attack teaching, this skill is hard formed only in a few days, and if they only take traditional mechanic training method that will create a boring studying climate which will then reduce the enthusiasm of trainers, day by day, they will lose confident. However, applying idea training will be different, it could let the trainers master the key points of forehand attack teaching, enforcing their confidence which could lead a positive attitude to take part in class teaching and learning. At last, such training could offer fine mental experience for trainers, to comfort the anxious state and be released, and then 
they will show the best of themselves in the actual training, improving their own sports performance [3].

\section{Applying Exploration in Idea Training of Forehand Attack Teaching}

This article is mainly take investigation to a part of students in a high school, using experimental inquiry method to analyze application of idea training in forehand attack teaching, and divide these students into two groups, which are experimental group with idea training and control group with traditional teaching method, the specific plan as following: first is for selection of the subjects, there are no obvious difference between two groups and before test, the propose and content of this experiment will be informed to these students, it is important to assure the corporation from students and the time in different group is simultaneous, avoiding other interrupted factors and make sure the contend is consistency. After testing, to take a consist test for these two groups, and the investigation shows that although two groups both grasp forehand attack, there are still obvious difference, the experimental group is better than control group, therefore, we could see that idea training is really helpful to improve sports skill of students. Besides, as for physical quality, height and weight in experimental group changes, although not very large but could see the improvement in general, which could prove that forehand attack can improve physical quality of students with effect [4]. And next, this article will explore from two aspects of application in designation of idea training and strategy, offering reference to related people.

\subsection{Designation of idea training of forehand attack training.}

According to the investigation, applying forehand attack teaching in table tennis could not only improve the sports skill of students but also physical quality, thus in actual teach, teachers should make reasonable design, when doing this, first thing conductors should to do is to train their idea ability which is the base to take further teaching of forehand attack in table tennis, if trainer are lack of corresponding performance, they could not apply such training method with effect. While training them, conductors should notice the difference of students such as factors in family, social, personal and so on, thus it teachers to employ suitable method to different students, and use targeted training will stimulate their learning enthusiasm and thus create a easy and light teaching climate for both sides. They then could let the trainers explore their mind to image and express, and students will be willing to take part in class activity [5]. Besides, it is needed to use video with movements for forehand attack teaching in table tennis which is good to form a visual image for trainers and thus make deeper impression, however, the important way is to watch video and then imitate movements it display, meanwhile, conductors should be the example of for students to enforce the effect. Next, it also needed to combine the imagination and imitation while designing idea training, the most key point of it is to trigger imagery ability of trainers, thus, during actual teach, the conductors should conduct trainers to combine imagery and imitation nicely, and teachers often use divided teaching to impart teaching movement of forehand attack, after explain one of actions, they will give several thinking time for students to image the taught movement just now, then to imitate, which could enforce impression of this movement [6]. And at last, while teaching the forehand attack in table tennis, teachers should notice the usage of language, that use different expression in same context will convey different feeling to others, thus there are two points for teachers to be aware of, first is to have enough teaching skill and knowledge of forehand attack, and show the key points of this method, only with it could students have better image. While taking forehand attack teaching in table tennis, teacher should emphasis on backswing, ball beating, waist-turning and so on, trying to avoid boringness, applying reasonable language to conduct trainers to operate imitation. Secondly, teachers should employ more encouraged words while taking actual forehand attack teaching, and because of difference between personality and learning ability, they could pay less attention to trains' sports performance which could express encourage to learners with good performance while reducing confident of bad performance by critics, therefore, teachers should notice their language whether suitable, for example, they use words like "you did very well" "you are the best " to students with better performance, and words like "I think you will do better next time" to those who are not good, 
these words could trigger interest of student to some extent, and thus improve the teaching quality [7].

\subsection{The applying strategy of forehand attack training in table tennis.}

There is only one right attack technology in forehand attack training, however, the wrong movements are various, there are mistakes as following: first is the wrongness in standing and direction of racket, in forehand attack, the beginners often take parallel standing and back-front standing, and while attacking the ball, they could not control the angle of racket that cause wrongness in direction of racket. Racket has two kinds, horizontal and pen-hold, using horizontal racket, the beginners will have situation like wrist internal rotation while have wrist adduction using pen-hold, both situation is easily to miss the ball, and as for racket, there will be situations as backward or forward with too much angle in beginners that leads to failure of attacking. Secondly, during attacking, the beginners also have mistakes in timing, part and position, many learner all think that it is wrong to beat the ball after it rebounds at apex of net, the right one is to beat the ball when it just at the top point, and the beating position is not back middle upper but back upper or back bottom, this is the key point of teaching which decide the accuracy of attacking. In forehand attack teaching in table tennis, the "triangle" theory is emphasized, which means players, ball and racket these three elements should make up a triangle, and once the position is wrong that will to some extent affect the beating quality. Right beating position is when the ball rebounding at the point that in front of breast and has one-elbow distance as high as apex net point [8]. At last, during taking table tennis training, it is very easily to have situation that the wrist could not turn or move slowly, while beating, the main force part is their wrist and once wrist could not turn or turn too much, it will affect the power and speed of beating, however, it is the hardest point and most difficult part that learners could not grasp at short time, and moving slowly is a common issue for trainers, in the actual training, there is no same two balls that every ball should be taken in position selecting and moving and slowly cause wrong beating. Besides, in actual forehand attack training, trainers also face many issue, therefore, teachers should pay attention to the implement strategy while designing [9].

First, it could apply positive and false contrast method in the forehand attack teaching, as for mistake that show vivid in outlook teacher could take contrast and demonstration to correct the wrongness. Secondly, teachers could apply idea training method of forehand attack in table tennis that leads students to observe their attacking movement through mirrors, and thus trigger imagery thinking of brain, to find out the beating problem. At last, is to offer video of excellent players to trainers, making contrast between them own and players thus realize the issue [9].

While teaching forehand attack in table tennis, teachers also need to make a scientific and reasonable design for training content and practical process. During teaching practice, teachers should consider to the actual situation of trainers and investigate the imagery ability of students to settle a reasonable training content, and thus improve the classing content gradually, while teaching, teachers also need to apply idea training into suitable training process, only with that could promote the whole effect of idea training, and in the process of forehand attack training, the implement of idea training could not operate without scientific and reasonable training content and practical process as well. First, teachers should adapt suitable method into idea training. Secondly, teachers should pay attention to demonstration of the key movement while impart forehand attack skill, and during it, students need to exercise to improve the normalization and thus they could apply idea training to recall the actions teachers just divided and explain, and correct the mistake exists in their own [10].

After teaching the corresponding skill of forehand attack to students, teachers should organize test to check the points where these trainers ever reached, and because trainers always at a highly centralized state while applying idea training in forehand attack, it will bring heave burden to them gradually, therefore, once do evaluation of them, there must leave several time for them to thinking. Do not be in a hurry to success. And during evaluation process, teachers should pay attention to the application of comprehensive skill among students, teachers could take measures like group compete, display with without tools to check the effect and quality of idea training [11]. 


\section{Conclusion}

To sum up, applying idea training of forehand attack teaching in table tennis could improve the teaching quality, and while teachers adapt the idea training method, they should pay attention to the students that help them arouse learning interest of forehand attack in table tennis, and in the actual teaching, teachers should grasp the designation of idea training and implement strategy of forehand attack teaching, only with that could idea training promote its whole effects.

\section{References}

[1] Wang Min, Liu Na. The influence of Imagery Training on Students' Forehand Attack in Table Tennis [J].Modern Vocational Education,2017(4):231. S

[2] Wang Qizhen, Gao Shilei, Xiong Weifei. Experimental Study on the Influence of Sports Imagery Training on Skill Operation of Table Tennis Beginners [J]. Contemporary Sports Technology, 2015(36):87-88.

[3] Sun Chengfu. Research on the Path Selection and Function of Visual Presentation Integrated into Table Tennis Training [J]. Journal of Heilongjiang University of Technology (Comprehensive Edition), 2017(12):20-23.

[4] Sun Chengfu, Lv Jixu. The Influence of Visual Imagery Training on Table Tennis Skills [J]. Journal of Harbin Institute of Physical Education, 2017(4):87-91.

[5] Tang Xingwang. The Application of Imagery Training in Table Tennis Teaching [J]. New Education Era, 2017(22):268.

[6] Xu Shuai. The Application of Idea Training in Table Tennis [J]. Rural Economy and Science-Technology, 2017(16):277.

[7] Ma Pengshuai,Li Xueqi, Bing Chuan. How to Apply the Imagery Training Method to the Forehand Attack Technique of Table Tennis [J]. Boxing \& Fight, 2017(14):51.

[8] Wei Yili, Bu Danran. An Empirical Study of Imagery Training in Badminton Training Of Women [J]. Journal of Hubei Sports Science, 2017(6):507-510.

[9] Li Meng. On the Idea Training Method in Table Tennis Teaching [J]. China Venture Capital, 2016(20):371-371.

[10] Yu Jucheng. Application of Analytical Imagery Training Method in Table Tennis Teaching [J].Bulletin of Sport Science \& Technology,2016(6):62-63.

[11] Xiao Xuefeng, Liu Chao, Zhao Yiping. Application of Idea Training Method in Ttable Tennis Serve Teaching [J]. Journal of Nanjing Sport Institute, 2016(3):93-97. 